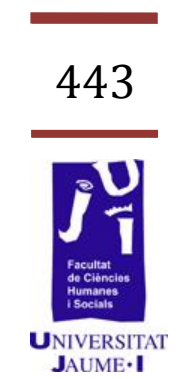

\title{
La «vida» del equipo cooperativo a través del Plan del Equipo
}

Santiago Traver Albalat al019644@alumail.uji.es 
Una herramienta didáctica muy potente para la enseñanzaaprendizaje del trabajo en equipo es el Plan del Equipo. Este plan consiste en una "declaración de intenciones" en la que los miembros de cada equipo hacen constar, para un período de tiempo determinado, el cargo que ejercerá cada componente, los objetivos del equipo y los compromisos personales. Se presentan los objetivos del equipo de los Planes del Equipo de uno de los equipos cooperativos de una aula de ocho alumnos/as de 5o de educación primaria. La implantación del aprendizaje cooperativo en el aula se ha realizado siguiendo las recomendaciones que se proponen en el Programa CA/AC ("Cooperar para aprender/Aprender a cooperar"), una programa didáctico para enseñar a aprender de manera cooperativa. La búsqueda constante de la mejora del trabajo en equipo a través del establecimiento de sucesivos Planes del Equipo muestra como a lo largo del curso los objetivos del equipo van articulando la "vida" de este equipo de aprendizaje cooperativo.

Palabras clave: aprendizaje cooperativo, atención a la diversidad, educación inclusiva, competencias básicas, equipo cooperativo, Plan del Equipo, educación primaria.

\section{Introducción}

A pesar de las recomendaciones de los organismos internacionales ni la política educativa, ni los centros, ni los profesores, ni los orientadores, ni las familias, ni ninguna institución de la sociedad civil se han preocupado de formar explícitamente en dos de los cuatro pilares propuestos: "aprender a ser" y "aprender a convivir juntos" (Delors, 1996). Según García (2011: 61) es evidente que las presiones ocasionadas por el puesto ocupado en el Informe PISA ${ }^{2}$ (Program for International Student Assessment) están teniendo como efecto que le demos excesiva importancia al conocer, al desarrollo de la dimensión cognitiva, y que nos despreocupemos de otras dimensiones básicas del ser humano (...) La finalidad de la educación se nos ha olvidado en este proceso, que es formar a personas, contribuir a su desarrollo integral y autónomo, a aumentar su capacidad para transformar la sociedad. Se trata de formar a personas que no sólo tengan recursos para adquirir conocimientos, sino que además manifiesten calidad en sus comportamientos; personas que sean capaces de desarrollar pensamiento crítico, pues los pensadores

\footnotetext{
${ }^{1}$ El Programa CA/AC es un programa didáctico para enseñar a aprender de manera cooperativa que explica cómo se puede implementar el aprendizaje cooperativo en las aulas (véase más información en: http://www.cife-ei-caac.com/).

${ }^{2}$ El Informe del Programa Internacional para la Evaluación de Estudiantes o Informe PISA por sus siglas en inglés (Program for International Student Assessment) se basa en el análisis del rendimiento de estudiantes a partir de unos exámenes mundiales que se realizan cada tres años y que tienen como fin la valoración internacional de los alumnos/as.
} 
críticos están comprometidos con la vida. En esta misma línea García y Candela (2010: 48) destacan que "aprender a vivir juntos" (...) se refiere básicamente aprender a conocer y respetar al otro diferente, a llegar a acuerdos, a promover proyectos comunes, a escuchar, a encontrar soluciones consensuadas por métodos no violentos. Sería importante practicar en la escuela el modelo democrático, consensuando normas sociales, fomentando la participación de los alumnos/as en todos aquellos aspectos relacionados con la convivencia (...) La escuela tiene la responsabilidad de educar a sus miembros procurando el desarrollo de actitudes y valores que los doten para ser ciudadanos conocedores de sus derechos y los de los demás, responsables en el cumplimiento de sus obligaciones, libres, cooperativos y tolerantes; es decir, ciudadanos capacitados para participar en la democracia.

Para Pujolàs (2008: 275-276) la estructuración cooperativa del aprendizaje en el aula es una manera de que nuestros alumnos y alumnas desarrollen a lo largo de su escolarización la competencia social y ciudadana que, según la Ley Orgánica de Educación, entre otros aspectos, consiste en comprender la realidad social, cooperar, convivir, ejercer la ciudadanía democrática en una sociedad plural, así como comprometerse a contribuir a su mejora.

De este modo, Riera (2010: 95) (...) define el aprendizaje cooperativo como el uso didáctico del trabajo en equipos reducidos dentro del aula, generalmente de composición heterogénea, utilizando una estructura de la actividad que asegure al máximo la participación equitativa de todos los miembros de un equipo y la interacción simultánea entre ellos, con la finalidad que aprendan -cada uno hasta el máximo de sus capacidades- los contenidos de las diferentes áreas y que aprendan, además, a trabajar en equipo.

Organizar la clase de forma cooperativa no es algo fácil; a veces aparece como un sueño pretender que en un grupo con tensiones, rivalidades, exclusiones, etc. los alumnos/as, en equipos reducidos, se ayuden unos a otros a aprender lo que el profesorado les enseña (Pujolàs y Lago, 2007: 356).

El programa CA $/ \mathrm{AC}^{3}$ (Cooperar para Aprender/Aprender a Cooperar) basado en el aprendizaje cooperativo, está formado por un conjunto de actuaciones encaminadas a enseñar al alumnado a trabajar en equipo. Dicho programa se estructura en tres ámbitos de intervención estrechamente relacionados (Pujolàs, 2008: 142):

1. El ámbito de intervención A (la cohesión del grupo): incluye todas las actuaciones relacionadas con la cohesión del grupo, para conseguir que, poco a poco, los alumnos y las alumnas de una clase tomen conciencia de grupo y se conviertan cada vez más en una pequeña comunidad de aprendizaje. Se trata de programar, dentro de la tutoría, una serie de

${ }^{3}$ En adelante Programa CA/AC. 
dinámicas de grupo ${ }^{4}$ que faciliten el "clima" y contribuyan a crear esta "conciencia de grupo" colectiva. Las actuaciones de este primer nivel de intervención no son exclusivas de una estructuración cooperativa del aprendizaje. Debemos considerarlas como actuaciones necesarias, si no imprescindibles, pero insuficientes para acabar estructurando de forma cooperativa las actividades educativas.

2. El ámbito de intervención $B$ (el trabajo en equipo como recurso para enseñar): abarca las actuaciones caracterizadas por la utilización del trabajo en equipo como recurso para enseñar, con el fin de que los alumnos/as, trabajando de esta manera, aprendan mejor los contenidos escolares porque se ayudan unos a otros. En este segundo nivel de intervención hay que dar un nuevo paso y utilizar, dentro del aula, el trabajo en equipos reducidos de alumnos/as como un recurso para asegurar la cooperación y la ayuda mutua y así aprender mejor los contenidos escolares (...) Para ello, la estructuras cooperativas ${ }^{5}$ de la actividad son imprescindibles puesto que garantizan la interacción entre todos los miembros de un equipo a la hora de trabajar juntos, porque los "obliga" a contar unos con otros.

3. El ámbito de intervención $\mathrm{C}$ (el trabajo en equipo como contenido a enseñar), finalmente, incluye las actuaciones encaminadas a enseñar a los alumnos y alumnas, de una forma explícita y sistemática, a trabajar en equipo, puesto que, además de un recurso para enseñar, el trabajo en equipo es un contenido que hay que enseñar. Enseñar a trabajar en equipo a nuestros alumnos/as consiste, básicamente, en ayudarles a especificar con claridad los objetivos que se proponen, las metas que tienen que alcanzar, enseñarles a organizarse como equipo para alcanzar estas metas (lo cual supone la distribución de distintos roles y responsabilidades dentro del equipo y la distribución de las distintas tareas, si se trata de hacer algo entre todos) y enseñarles, practicándolas, las habilidades sociales imprescindibles para trabajar en grupos reducidos. A medida que trabajan juntos, se conocen más a fondo y se hacen más amigos/as, lo cual va incrementando en ellos lo que se denomina interdependencia positiva de identidad. Para incrementar esta identidad se puede utilitzar el Cuaderno del Equipo. El Cuaderno del Equipo es un cuaderno donde los equipos deben hacer constar: su nombre y su logotipo, los nombres de sus componentes, los cargos y funciones, las normas de funcionamiento, los "diarios de las sesiones", los Planes del Equipo y las revisiones periódicas del grupo.

Así pues, según Pujolàs y Lago (2007) para introducir, de forma paulatina, una estructuración cooperativa del aprendizaje en el aula debemos intervenir sobre los tres niveles o ámbitos de intervención mencionados. No se trata de tres pasos sucesivos, que hay que realizar

\footnotetext{
${ }^{4}$ Véanse descritas en Pujolàs (2008: 165-183).

${ }^{5}$ Véanse descritas en Pujolàs (2008: 199-212).
} 
uno tras otro, sino de tres niveles de intervención paralelos que introducimos de forma sucesiva pero que acabamos trabajando en ellos de forma prácticamente simultánea. Es decir, las intervenciones relacionadas con el segundo nivel no sustituyen a las del primer nivel, ni las del tercer nivel sustituyen a las del segundo, sino que después de haber intervenido en el primer nivel, y sin dejar de llevar a cabo sucesivas actuaciones propias de este nivel, introducimos actuaciones del segundo nivel $y$, sin dejar éstas, introducimos finalmente las actuaciones propias del tercer nivel.

\section{Finalidad}

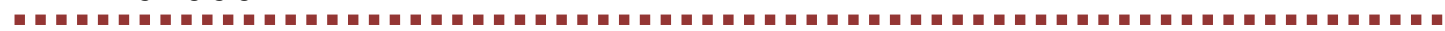

La finalidad que se pretende conseguir con la realización de este trabajo es la siguiente:

Ofrecer una aproximación de cómo a lo largo de un curso escolar se va articulando la "vida" de un equipo de aprendizaje cooperativo con los objetivos que el equipo se propone a través del establecimiento de sucesivos Planes del Equipo y sus correspondientes revisiones periódicas.

\section{Contextualización}

La experiencia se ha llevado a cabo durante el curso escolar 20092010 en una aula de 5o de primaria de un colegio público, del ámbito rural, de la provincia de Castellón. Se trata de una aula de 8 alumnos/as distribuida en dos equipos de base heterogéneos en la que cada equipo está formado por 4 alumnos/as.

La introducción del aprendizaje cooperativo en el aula se ha realizado siguiendo el modelo que se propone en el Programa CA/AC. Cabe destacar, que los alumnos/as de esta aula siempre habían trabajado con una estructuración competitiva y/o individualista del aprendizaje y la metodología cooperativa se presentó como una novedad. A lo largo del curso escolar se ha trabajado cooperativamente en las áreas de matemáticas, valenciano y conocimiento del medio, en el resto de áreas se ha utilizado una estructuración del aprendizaje individualista y/o competitiva.

De este modo, a continuación, nos centraremos en el ámbito de intervención $\mathrm{C}$ del programa, el trabajo en equipo como contenido a enseñar, ya que se trata del objetivo principal de este trabajo. 


\section{El trabajo en equipo como contenido a enseñar}

Según Pujolàs (2012: 89) el trabajo en equipo no es sólo un recurso para enseñar (como hemos destacado en el ámbito de intervención B), sino también un contenido más que debemos enseñar (como se pretende destacar en el ámbito de intervención C). Si esto es así, debemos enseñar este contenido, como mínimo, de una forma tan sistemática y persistente como enseñamos los demás contenidos de las distintas áreas.

Como explica este mismo autor, en la práctica, los pasos para la organización interna de los equipos incluyen los siguientes elementos:

- La elección de un nombre y un logotipo del equipo.

- El recordatorio constante de los objetivos que persigue el equipo.

- La determinación de las normas de funcionamiento y de convivencia.

- La organización interna del equipo con la determinación y distribución de los cargos a ejercer dentro de él, con la especificación de las funciones propias de cada cargo.

- La búsqueda constante de la mejora del equipo a través del establecimiento de sucesivos Planes del Equipo -y sus correspondientes revisiones- con los objetivos que el equipo se propone para un período de tiempo determinado y los compromisos que los miembros del equipo contraen para conseguir estos objetivos.

- La articulación de la "vida" y la "historia" del equipo a través del Cuaderno del Equipo.

Todos estos elementos se van desarrollando paralelamente a las sesiones de clase de las distintas áreas en las cuales los alumnos y las alumnas trabajan de forma cooperativa.

Ahora bien, el medio fundamental que propone el ámbito de intervención C (el trabajo en equipo como contenido a enseñar) del programa para que los alumnos y las alumnas aprendan a trabajar en equipo es el Plan del Equipo (...) Se trata de una "declaración de intenciones", de un "proyecto", una previsión de lo que se proponen conseguir, tener en cuenta o fijarse de una forma especial durante el período de vigencia del plan (generalmente, quince días o un mes) (...) Los elementos fundamentales de un Plan del Equipo -y que, a su vez, sirven para regular el funcionamiento de los equipos- son los siguientes:

- Los objetivos del equipo: los objetivos que se fijan como equipo (no son los objetivos didácticos de la materia sobre la que están trabajando en equipo $\left.{ }^{6}\right)$. Al tratarse de equipos de aprendizaje y cooperativos, dos objetivos son fijos y comunes a todos los equipos: aprender cada uno al máximo y ayudarse a aprender. Otros objetivos son variables y pueden ser propios de un equipo determinado. De este modo, pueden proponerse como objetivos mejorar algún aspecto, especialmente conflictivo o poco

${ }^{6}$ En todo caso, pueden considerarse los "objetivos didácticos" del "contenido" "trabajar en equipo". 
conseguido, de su funcionamiento como equipo: estar dispuestos a ayudar, darse ánimos mutuamente, etc.

- Los cargos del equipo: los cargos que tendrán que distribuir y ejercer dentro del equipo, teniendo en cuenta que:

-El nombre de los cargos puede variar en cada equipo y depende de la edad o de la etapa educativa. Por ejemplo, responsable o coodinador, ayudante del responsable o coordinador, portavoz, secretario, responsable del material, etc.

- Hay que operativizar al máximo los distintos roles o cargos, indicando las funciones propias de cada cargo. A modo de ejemplo, una de la funciones del rol de coordinador podría ser "animar a los miembros del grupo" y del secretario "custodiar el cuaderno del equipo", entre otras.

- Caba miembro del equipo debe ejercer un cargo.

-Los cargos son rotativos.

-Los alumnos/as deben exigirse mutuamente ejercer con responsabilidad las funciones propias de su cargo.

- Los compromisos personales: en este apartado del Plan del Equipo cada estudiente especifica a qué se compromete personalmente para mejorar el funcionamiento del equipo. Es una forma de educar la responsabilidad de cada uno, intentando mejorar en algo su comportamiento por el bien del equipo.

\section{PLAN DEL EQUIPO}

Período:

\section{CARGO DENTRO DEL EQUIPO}

\section{Cargo:}

Coordinador/a

Ayudante del coordinador

Secretario/a

Responsable del material

\section{OBJETIVOS DEL EQUIPO}

3. COMPROMISOS PERSONALES

Realizado por:

Logotipo del equipo: 
Hacia el final del período de vigencia de un Plan del Equipo determinado, los miembros del equipo deben reunirse para evaluar el plan, para revisar su funcionamiento durante este período de tiempo, identificar lo que hacen especialmente bien y los aspectos que deben mejorar, y determinar, a partir de la valoración, los objetivos y los compromisos personales del siguiente Plan del Equipo. En este sentido, y desde la práctica, cabe destacar que para que la revisión del Plan del Equipo sea realmente democrática todos los miembros del equipo tienen que participar en las valoraciones de los diferentes apartados del plan y, además, la valoración final del equipo de cada uno de los apartados del Plan del Equipo debe recoger las aportaciones de todos los miembros del equipo.

Tabla 2. Modelo de revisión periódica del Plan del Equipo

\section{REVISIÓN PERIÓDICA DEL PLAN DEL EQUIPO}

Período:

Logotipo del equipo:

\section{1. ¿HEMOS EJERCIDO CORRECTAMENTE NUESTRO CARGO?}

Cargo:

Necesita mejorar porque...

Bien porque...

Coordinador/a

Ayudante del coordinador

Secretario/a

Responsable del material

\section{2. ¿HEMOS ALCANZADO LOS OBJETIVOS DEL EQUIPO?}

OBJECTIVOS PROPUESTOS SI NO Tenemos que mejorar porque...

\section{3. ¿HA CUMPLIDO CADA UNO SU COMPROMISO PERSONAL?}

Nombre Compromiso personal Necesita mejorar Bien Muy bien

Esta forma de proceder requiere que los equipos de base sean estables, que sus componentes permanezcan juntos un tiempo suficiente como para llevar a cabo distintos Planes del Equipo, de modo que tengan la 
oportunidad de cambiar lo que no hacen bien y consolidar lo que hacen adecuadamente.

Poco a poco, planificación tras planificación, el funcionamiento de los equipos va mejorando porque van "interiorizando" y "consolidando" las habilidades sociales relacionadas con el trabajo en estos pequeños grupos, las relaciones entre los participantes se hacen más positivas porque se conocen más, se respetan más y son más amigos/as... De esta manera, paulatinamente, aprenden a trabajar en equipo porque adquieren las habilidad de organizarse mejor y de funcionar mejor como equipos de aprendizaje cooperativo.

\section{Metodología científica}

Ubicado en el paradigma de investigación educativa interpretativo, se trata de un estudio de caso naturalista (Stake, 1998: 15) con un enfoque cualitativo de corte etnográfico, porque el grupo seleccionado es una unidad formada naturalmente, un aula de primaria que ya existía como tal en el colegio.

La muestra se define como fortuita o accidental. Al ser el propio investigador el tutor del aula ha seleccionado su propia clase porque la tenía más a mano.

Para analizar los datos se realizó un análisis del contenido por trimestres de los objetivos de los Planes del Equipo del equipo cooperativo en cuestión. Se hizo por trimestres teniendo en cuenta los tres trimestres en los que se divide el curso escolar. Siguiendo a Stake (1998) en el estudio intrínseco de casos, el investigador secuencia la acción, categoriza las propiedades, y hace recuentos para sumarlos de forma intuitiva (...) Normalmente, se intenta dedicar la mayor parte del tiempo a la interpretación directa (Stake, 1998: 71).

\section{Resultados}

Se presentan los objetivos del equipo de todos los Planes del equipo de uno de los equipos cooperativos del aula a lo largo de todo el curso escolar. En primer lugar, se pueden observar los objetivos del equipo de los dos Planes del equipo realizados durante del primer trimestre de 5o de primaria:

Tabla 3. Objetivos del equipo del primer Plan del Equipo del primer trimestre

\begin{tabular}{|l|}
\hline \multicolumn{2}{|c|}{ OBJETIVOS DEL PLAN DEL EQUIPO } \\
\hline \multicolumn{1}{|c|}{ Período: 3/11/2009 hasta 30/11/2009 } \\
\hline -Hablar flojo \\
\hline -No armar alboroto \\
\hline -Levantar el brazo para hablar y esperar el turno \\
\hline -No correr por dentro de la clase \\
\hline
\end{tabular}


Tabla 4. Objetivos del equipo del segundo Plan del Equipo del primer trimestre

OBJETIVOS DEL PLAN DEL EQUIPO

\begin{tabular}{|l|}
\hline \multicolumn{1}{|c|}{ OBJETIVOS DEL PLAN DEL EQUIPO } \\
\hline Período: $1 / 12 / 2009$ hasta $21 / 12 / 2009$ \\
\hline -Hablar menos \\
\hline - Cumplir las normas \\
\hline -No correr por dentro de la clase \\
\hline
\end{tabular}

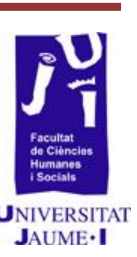

A continuación, se presentan los objetivos del equipo de los cuatro Planes del equipo realizados a lo largo del segundo trimestre del curso escolar.

Tabla 5. Objetivos del equipo del primer Plan del Equipo del segundo trimestre

\begin{tabular}{|l|}
\hline \multicolumn{2}{|c|}{ OBJETIVOS DEL PLAN DEL EQUIPO } \\
\hline \multicolumn{1}{|c|}{ Período: 10/01/2010 hasta 1/02/2010 } \\
\hline -Pedir ayuda \\
\hline -Dar ayuda \\
\hline -Felicitar a los compañeros \\
\hline -Cumplir los cargos bien \\
\hline
\end{tabular}

Tabla 6. Objetivos del equipo del segundo Plan del Equipo del segundo trimestre

\begin{tabular}{|l|}
\hline \multicolumn{1}{|c|}{ OBJETIVOS DEL PLAN DEL EQUIPO } \\
\hline Período: $1 / 02 / 2010$ hasta 20/02/2010 \\
\hline -Cumplir las normas de clase \\
\hline -Cumplir los cargos bien \\
\hline -Preocuparse porque todos aprendan \\
\hline -Cumplir los cargos bien \\
\hline
\end{tabular}

Tabla 7. Objetivos del equipo del tercer Plan del Equipo del segundo trimestre

\begin{tabular}{|l|}
\hline \multicolumn{1}{|c|}{ OBJETIVOS DEL PLAN DEL EQUIPO } \\
\hline \multicolumn{1}{|c|}{ Período: 22/02/2010 hasta 12/03/2010 } \\
\hline -Hablar cuando toca \\
\hline -No molestar a los compañeros \\
\hline -Felicitar al equipo \\
\hline
\end{tabular}

Tabla 8. Objetivos del equipo del cuarto Plan del Equipo del segundo trimestre

\begin{tabular}{|l|}
\hline OBJETIVOS DEL PLAN DEL EQUIPO \\
\hline \\
\hline -Felicitar al equipo \\
\hline
\end{tabular}


-Hablar de cosas de dentro de la escuela

-Levantar el brazo para hablar

En tercer lugar, y para terminar este apartado de resultados, se pueden observar los objetivos de los Planes del equipo que se plantearon los alumnos/as de este equipo hasta final de curso.

Tabla 9. Objetivos del equipo del primer Plan del Equipo del tercer trimestre

\begin{tabular}{|l|}
\hline \multicolumn{2}{|c|}{ OBJETIVOS DEL PLAN DEL EQUIPO } \\
\hline \multicolumn{1}{|c|}{ Período: $19 / 04 / 2010$ hasta 5/05/2010 } \\
\hline -Hablar de cosas de dentro de la escuela \\
\hline -Levantar el brazo para hablar \\
\hline -Estar atentos \\
\hline
\end{tabular}

Tabla 10. Objetivos del equipo del segundo Plan del Equipo del tercer trimestre

\begin{tabular}{|l|}
\hline \multicolumn{2}{|c|}{ OBJETIVOS DEL PLAN DEL EQUIPO } \\
\hline \multicolumn{1}{|c|}{ Período: 5/05/2010 hasta 24/05/2010 } \\
\hline -Estar atentos \\
\hline -Levantar el brazo para hablar \\
\hline
\end{tabular}

Tabla 11: Objetivos del equipo del tercer Plan del Equipo del tercer trimestre

\begin{tabular}{|l|}
\hline \multicolumn{1}{|c|}{ OBJETIVOS DEL PLAN DEL EQUIPO } \\
\hline \multicolumn{1}{|c|}{ Período: 24/05/2010 hasta final de curso } \\
\hline -No hablar de cosas de fuera de la escuela \\
\hline -Levantar el brazo para hablar \\
\hline -No molestar al equipo \\
\hline
\end{tabular}

\section{Discusión}

Siguiendo a Pujolàs y Lago (2012: 25-26), en la actualidad, más de 300 centros de Educación Infantil, Primaria y Secundaria de toda España aplican el Programa CA/AC a partir de un proceso de formación y asesoramiento, estructurado en tres grandes etapas: introducción, generalización y consolidación. La Universitat de Vic celebró los días 30 de junio y 1 de julio de 2011 el I Simposio sobre Aprendizaje Cooperativo, al que asistieron 224 maestros de Educación Infantil y Primaria, profesores de Secundaria y asesores de diferentes centros de profesores (...), y del que surgen algunas reflexiones sobre el programa, en el que todos ellos participan (...) Se pone de manifiesto que los tres ámbitos de intervención del Programa CA/AC están estrechamente relacionados y cualquier cambio o incidencia sobre uno repercute en los otros dos. Por otra parte, las actuaciones en estos tres ámbitos suponen cambios importantes, substanciales, en la forma de enseñar del profesorado y en la 
organización del centro, que implican superar inercias y formas de proceder muy enraizadas en los docentes, el alumnado, los centros y las familias. De ahí la necesidad de acumular una cierta cantidad de experiencias de trabajo en equipo, de notable calidad, para empezar a tener evidencias sobre la mejora de los aprendizajes de los alumnos y de su rendimiento (...) Ahora bien, en relación al tercer ámbito de intervención del programa, el trabajo en equipo como contenido a enseñar, se destaca la complementariedad de los instrumentos utilizados para que los alumnos y alumnas aprendan a trabajar en equipo, y la conexión que existe entre ellos: la planificación del trabajo, las valoraciones intermedias o el seguimiento de las sucesivas planificaciones, y la reflexión sobre el historial del equipo recogida en un cuaderno a tal efecto, para tomar conciencia de la evolución del grupo y de los cambios acontecidos. En este sentido, aportamos, a continuación, nuestro granito de arena a dicho ámbito de intervención (el trabajo en equipo como contenido a enseñar), a la luz de los resultados obtenidos en los Planes del Equipo de un equipo de aprendizaje cooperativo a lo largo del curso de 5 o de educación primaria.

Durante el primer trimestre del curso escolar podemos observar que los objetivos de ambos Planes del Equipo hacen referencia a las dificultades de los miembros del equipo en aspectos comunicativos, es decir, respetar el turno de palabra (no hablar más de un alumno/a a la vez) y tener cuidado del tono de la voz (no levantar el tono de la voz ni gritar). A este respecto, nos remitimos a los objetivos del Plan del Equipo: "hablar flojo", "no armar alboroto", "hablar menos" y "levantar el brazo para hablar y esperar el turno". Por tanto, se puede afirmar que abordar el trabajo de las competencias de carácter socioemocional en el aula se presenta como necesario para conseguir un trabajo óptimo durante el trabajo en equipos reducidos. Desde el GRAD ${ }^{7}$ (2012: 38) (Grup de Recerca en Atenció a la Diversitat de la Universidad de Vic) se destaca que el trabajo por competencias debe promover un trabajo integral en el aula y que la metodología cooperativa es una manera de llegar a más de una competencia simultáneamente, sobre todo a aquellas de carácter socioemocional, que constituyen la base para el aprendizaje.

De los objetivos del equipo de los Planes del Equipo del segundo trimestre, en primer lugar, se observa que los miembros del equipo todavía presentan algunas dificultades en ciertas competencias socioemocionales a lo largo de todo el trimestre. Nos referimos, de nuevo, a las competencias socioemocionales relacionadas con aspectos comunicativos. En este sentido, se plantean como objetivos: "levantar el brazo para hablar", "hablar de cosas de dentro de la escuela" y "no molestar a los compañeros". También se pueden constatar objetivos referidos a las dificultades en el cumplimiento de los cargos, ya que en los dos primeros Planes del Equipo de este trimestre aparecen objetivos planteados en esta dirección. No obstante, y ya des del principio de segundo trimestre y lo largo de él, se considera muy relevante, por una parte, la aparición de objetivos del equipo relacionados con las dificultades de los miembros del equipo para darse apoyo emocional, nos estamos refiriendo al objetivo del Plan del Equipo "felicitar al equipo". Este objetivo aparece en tres de los cuatro Planes del Equipo del segundo trimestre del curso escolar. Por otra parte, también consideramos importante la aparición de los objetivos "dar ayuda", "pedir

${ }^{7}$ EI GRAD -grupo de investigación consolidado- es un grupo de investigación interdisciplinario formado por profesorado de diferentes departamentos de la Universidad de Vic, así como también por diferentes agentes del ámbito educativo (maestros/as, psicopedagogos/as, psicólogos/as y pedagogos/as). 
ayuda" y "preocuparse porque todos aprendan" en los dos primeros Planes del Equipo de este trimestre, entendiendo las dificultades que se observaron en su cumplimiento al iniciar el segundo trimestre del curso.

Los objetivos de los Planes del Equipo del tercer trimestre del curso escolar muestran, de manera clara, que a lo largo del curso se mantienen las dificultades en aspectos comunicativos entre los miembros del equipo. Buena muestra de ella son los siguientes objetivos: "levantar el brazo para hablar", "estar atentos", "no hablar de cosas de fuera del colegio" y "no molestar al equipo".

Finalmente, se hace necesario retomar la finalidad de este trabajo, para concluir que la "vida" de este equipo de aprendizaje cooperativo, articulada a través de los objetivos de los Planes del Equipo que sus miembros se plantearon a lo largo del curso de 50 de primaria, queda definida a grandes rasgos por dos aspectos fundamentalmente. En primer lugar, la estabilidad en el tiempo de las dificultades en las competencias socioemocionales relacionadas con aspectos comunicativos. $Y$ en segundo lugar, la mejora a lo largo del curso de las dificultades encontradas en lo referente al apoyo académico y emocional, ya que en los objetivos de los Planes del Equipo del tercer trimestre no emergieron objetivos en este sentido.

En definitiva, se ha ofrecido una aproximación a la "vida" de un equipo de aprendizaje cooperativo a través de los objetivos del equipo de los Planes del equipo realizados a lo largo de un curso escolar.

\section{Referencias bibliográficas}

García, R. y CANDELA, M.I. (2010). "La educación para la vida: el reto de aprender a ser y a vivir juntos en la Educación Secundaria". Revista Edetania, núm. 38, pp. 41-56.

García, R. (2011). "El profesorado de secundaria y su compromiso con la ética docente". Revista Edetania, núm. 39, pp. 57-70.

Grup de Recerca sobre Atenció a la Diversitat (GRAD) (2012). "Retos de futuro". Cuadernos de pedagogía, núm. 428, pp. 37-40.

MINISTERIO DE EDUCACIÓN Y CIENCIA. R.D. 2/2006 de 3 de mayo. LOE (Ley Orgánica de educación). BOE núm.106.

PUJOLÀS, P.; LAGO, J.R. (2007). "La organización cooperativa de la actividad educativa", pp. 349-392. Dentro BonALS, J. Manual de asesoramiento psicopedagógico. Barcelona: Graó.

PujolÀs, P. (2008). 9 ideas clave. Aprendizaje cooperativo. Graó: Barcelona.

PujolÀs, P. (2012). "La implantación del aprendizaje cooperativo en las aulas", pp. 77-104. Dentro Torrego, J.C. y Negro, A. Aprendizaje cooperativo en las aulas. Fundamentos y recursos para su implantación. Madrid: Alianza Editorial. 
PUjolÀs, P. y LAGO, J.R. (2012). "Un programa para cooperar y aprender". Cuadernos de Pedagogía, núm. 428, pp. 24-26.

RierA I ROMERO. (2010). Tesis doctoral: "Cooperar per aprendre/Aprendre a cooperar" (Programa CA/AC). Avaluació d'un programa didàctic per ensenyar a aprendre de manera cooperativa, pp. 95, 2010.

STAKE, R.E. (1998). Investigación con estudio de casos. Madrid: Morata

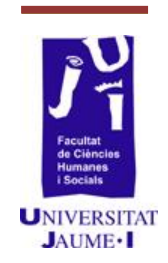

